\title{
Cutaneous Periumbilical Purpura in Disseminated Strongyloidiasis in Cancer Patients: A Pathognomonic Feature of Potentially Lethal Disease?
}

\author{
Jorge I. F. Salluh ${ }^{1,2,3}$, Fernando A. Bozza ${ }^{4}$, \\ Tatiana S. Pinto ${ }^{1}$, Luisa Toscano ${ }^{1}$, \\ Peter F. Weller ${ }^{5}$ and Marcio Soares ${ }^{1}$
}

\author{
Intensive Care Unit, National Institute of Cancer (INCA) ${ }^{1}$, School of \\ Medicine - Federal University of Rio de Janeiro ${ }^{2}$, Intensive Care Unit, \\ Barra D’Or Hospital ${ }^{3}$, Clementino Fraga Filho Hospital - Federal \\ University of Rio de Janeiro ${ }^{4}$; Rio de Janeiro, RJ, Brazil; Harvard \\ Thorndike Laboratories and the Department of Medicine, Beth Israel \\ Deaconess Medical Center, Harvard Medical School ${ }^{5}$, Boston, MA, USA
}

\begin{abstract}
Cutaneous manifestations in disseminated strongyloidiasis are infrequent but should raise the suspicion for its diagnosis. We retrospectively evaluated the charts of six patients with cancer and a proven diagnosis of disseminated strongyloidiasis. All patients had received prophylaxis with albendazole before starting antineoplastic therapy, which included high-dose steroids. They presented with septic shock, acute respiratory failure and characteristic purpuric periumbilical skin lesions. Strongyloides larvae were identified in tracheal aspirates $(n=5)$, gastric aspirates $(n=4)$, lung $(n=2)$ and skin biopsies $(n=2)$. All patients died despite antihelminthic therapy and intensive care support. Key Words: Sepsis, purpura, cancer, disseminated strongyloidiasis, corticosteroids.
\end{abstract}

Immunocompromised patients may develop disseminated strongyloidiasis, which results in high mortality rates [1,2]. Therefore, clinical suspicion is essential for early diagnosis and improvement in survival. Cutaneous manifestations in disseminated strongyloidiasis are infrequent, but they may be a valuable diagnostic sign [3-7]. We report six immunosuppressed cancer patients with proven diagnosis of disseminated strongyloidiasis presenting with periumbilical purpura and septic shock.

\section{Material and Methods}

This study was conducted at the Instituto Nacional de Câncer (INCA), Rio de Janeiro, Brazil, a public

Received on 8 July 2005; revised 30 August 2005.

Address for correspondence: Dr. Jorge I. F. Salluh. Instituto Nacional de Câncer. Intensive Care Unit - $10^{\circ}$ Andar. Praça Cruz Vermelha, 23 - $10^{\circ}$ Andar. Rio de Janeiro - RJ, Brazil. Zip code: 20230-130. Phone: +55219156 9038 / +55212506 6120 Fax: +55 21 2294.8620. E-mail: jorgesalluh@yahoo.com.br.

The Brazilian Journal of Infectious Diseases 2005;9(5):419-424 (C) 2005 by The Brazilian Journal of Infectious Diseases and Contexto Publishing. All rights reserved. hospital for referral of cancer patients. From February 2002 to September 2003, six patients with disseminated strongyloidiasis were admitted to the intensive care unit (ICU). Their charts and records were retrospectively evaluated.

\section{Case Reports}

We describe six cases of immunosuppressed cancer patients with proven diagnoses of disseminated strongyloidiasis presenting with periumbilical purpura. All patients received antihelminthic prophylaxis with albendazole (400 mg BID/3 days) prior to the antineoplastic therapies, which included high doses of steroids, chemotherapy, and radiation therapy. All patients had been admitted to the ICU after the development of acute respiratory failure (ARF) and shock . Patients had periumbilical purpura that was noticed soon after admission to the ICU; this spread in a centrifugally throughout the abdomen and groin. Their clinical characteristics are described in Table 1 . The diagnosis of disseminated strongyloidiasis was made in all six patients, as Strongyloides stercoralis was 
Table 1. Characteristics of six cancer patients with disseminated strongyloidiasis*

\begin{tabular}{lc}
\hline Characteristics & \\
\hline Gender & 1 \\
Female & 5 \\
Male & $57.5(27-69)$ \\
Age (years) & \\
Type of cancer & 2 \\
$\quad$ Hematologic malignancy & \\
$\quad$ Solid tumor & 4 \\
Clinical features at ICU admission & 6 \\
$\quad$ Septic shock & 6 \\
$\quad$ Acute respiratory failure & 5 \\
$\quad$ Abdominal distension & 6 \\
Immunosuppression & 3 \\
$\quad$ Corticosteroids & 2 \\
Chemotherapy & $30(10-210)$ \\
$\quad$ Radiation therapy & 6 \\
Duration of immunosuppressive therapy (days) & 6 \\
Antihelminthic therapy & 2 \\
Thiabendazole & 6 \\
Ivermectin & 6 \\
Albendazole prophylaxis & $11.5(5-23)$ \\
Multiple organ failure in the ICU & 6 \\
ICU length of stay (days) & \\
Death in the ICU & \\
\hline CU & \\
\hline
\end{tabular}

ICU=Intensive care unit. * Results in number and median (range).

Table 2. Laboratory and microbiological data of six cancer patients with disseminated strongyloidiasis*

\begin{tabular}{lc}
\hline Characteristics & \\
\hline Eosinophilia & 0 \\
$\quad$ Yes & 6 \\
No & \\
Total platelet count $/ \mathrm{mm}^{3}$ at diagnosis & 5 \\
Site of Strongyloides stercoralis identification & 4 \\
$\quad$ Tracheal aspirate & 2 \\
Gastric aspirate & 2 \\
Skin biopsy & 1 \\
Lung biopsy & \\
BSI & 2 \\
Gram negative blood stream infection & 4 \\
Yes & \\
No & \\
\hline
\end{tabular}

* Results in number and median (range). 
Figure 1. Purpuric and petechial rash involving the periumbilical area, abdomen and thighs in a cancer patient with disseminated strongyloidiasis.

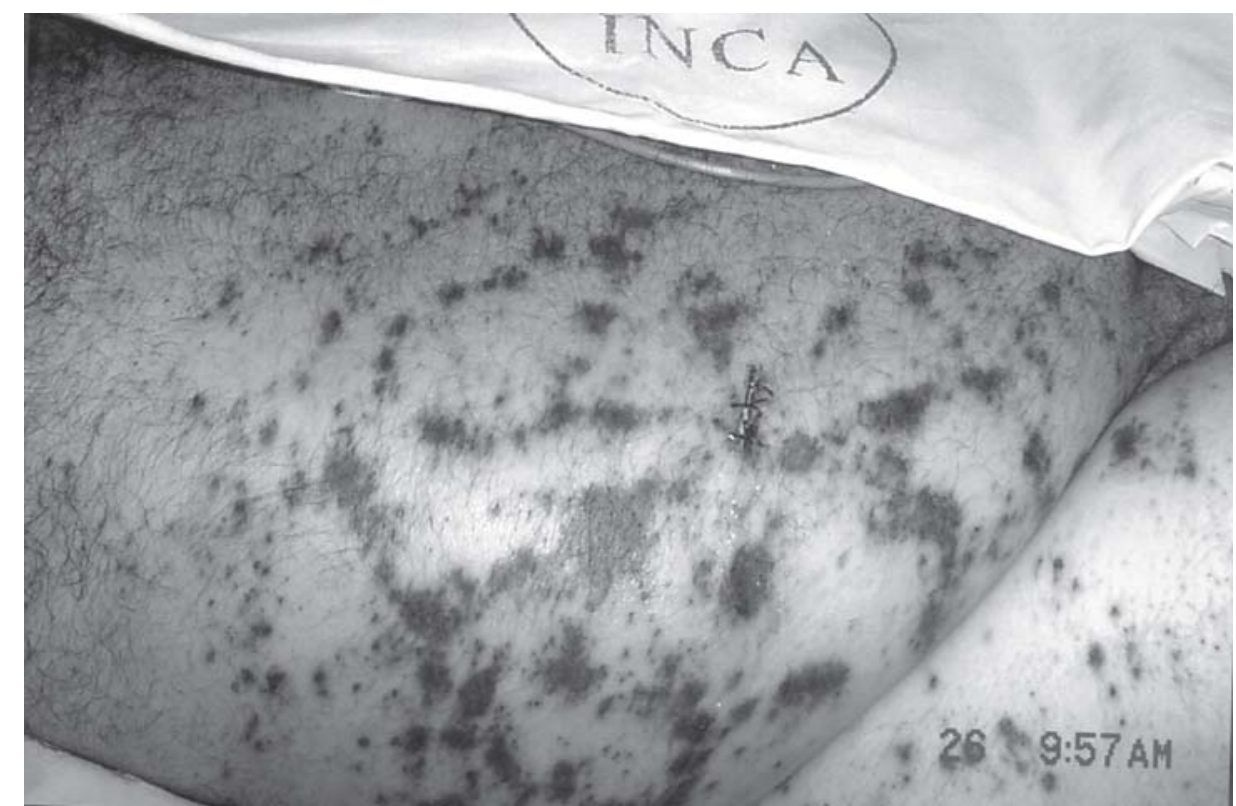

Figure 2. Section of skin biopsy with filariform larva of Strongyloides stercoralis between collagen bundles in the dermis of a cancer patient with disseminated strongyloidiasis. There was no inflammatory reaction.

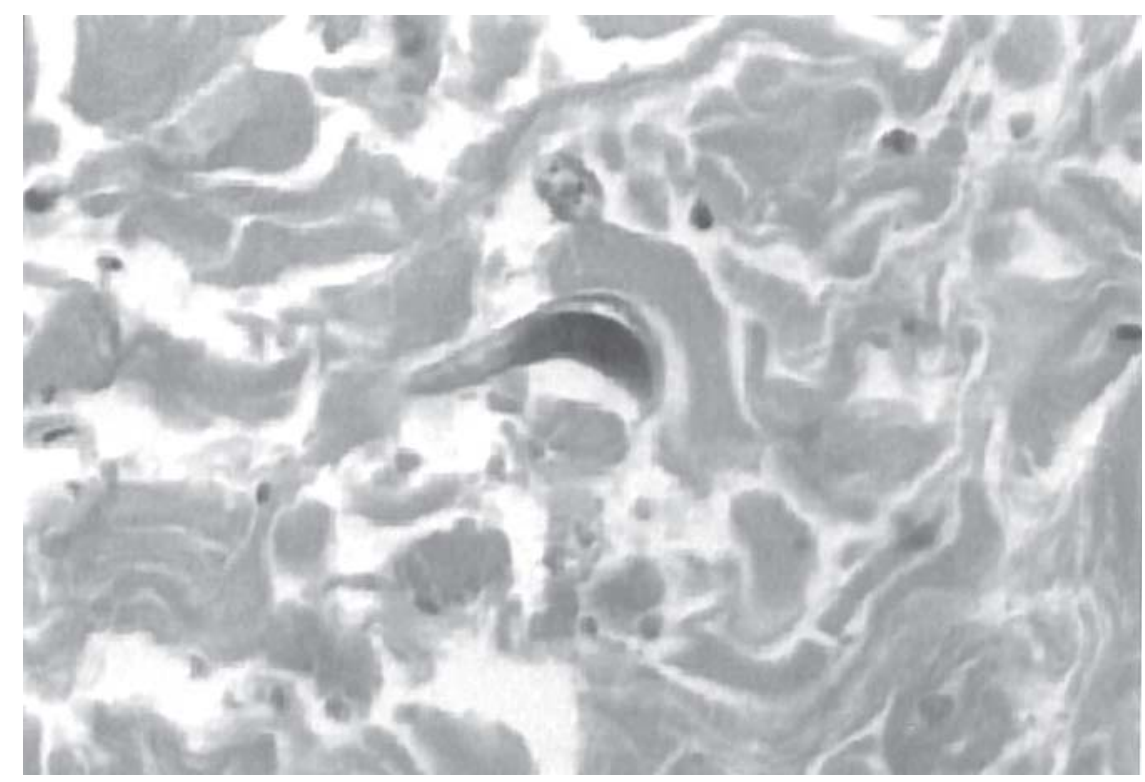


recovered in several types of biological samples, including tracheal aspirates, gastric aspirates, stool examination, lung biopsy and skin biopsy (see Table 2 for microbiological and laboratory data). Eosinophilia was not found in any of the patients, and platelet count was abnormal in half of the patients.

Despite full intensive care unit support, which included mechanical ventilation, use of vasopressors, broad-spectrum antibiotics, hemodialysis and antihelminthic therapy with thiabendazole (associated with ivermectin in two cases), all patients subsequently developed acute respiratory distress syndrome (ARDS), overt septic shock, and finally multiple organ failure (MOF) and death.

\section{Discussion}

We examined six cancer patients with septic shock, ARF and purpuric periumbilical cutaneous lesions, as manifestations of disseminated strongyloidiasis. All patients received prophylaxis with albendazole prior to the antineoplastic therapy. Despite the diagnosis of disseminated strongyloidiasis and antihelminthic therapy with thiabendazole ( \pm ivermectin), all patients died within the first three weeks of ICU admission due to MOF.

Unusually, strongyloidiasis may cause dermatological lesions. Petechial and purpuric lesions on the lower abdomen and thighs have been documented in the literature [6,7]. Purpura is attributed to the invasion of the dermis by larvae that migrate through the vessel walls [8]. It has been proposed that the periumbilical distribution may result from retrograde venous migration. Additionally, larvae may penetrate into the skin from the abdominal cavity, following migration through the large-bowel wall. In our series, all patients had rapidly evolving periumbilical purpura that extended in a centrifugal fashion throughout the abdomen and thighs. Two out of three skin biopsies were positive for $S$. stercoralis larvae. Diagnostic procedures, such as examination of stools, gastric or duodenal aspirations, tracheal secretions and bronchoalveolar lavage are useful for the confirmation of the diagnosis $[1,9,10]$. Four of our patients had positive gastric aspirate specimens and five had larvae in tracheal secretions. In two cases, a lung biopsy revealed the presence of larvae.

All of our patients had taken high doses of steroids and had at least one additional risk factor for strongyloidiasis. Two patients have received chemotherapy, three received radiation therapy and two had hematological malignancies. In addition, corticosteroids cause depression of cell-mediated immunity and are postulated to be a direct stimulator of Strongyloides [11,12]. The heavy burden of immunosuppression probably contributed to the overwhelming infection. Penetration of larvae through tissues facilitates the dissemination of intestinal bacteria, causing sepsis $[10,13]$. Hence, it was not unexpected that patients had clinical features of septic shock and two of them had blood cultures positive for Gram-negative bacteria.

It is well known that the most common worldwide cause of eosinophilia is helminthic infection [1]. However, none of our patients had eosinophilia at the time of diagnosis. This might have occurred because of the use of steroids, sepsis and leukopenia. Strongyloides stercoralis prophylaxis in immunosuppressed patients is still controversial [14]. In our study, all patients received previous anthelminthic prophylaxis with albendazole. This is an approach that has been suggested to diminish mortality due to strongyloidiasis [15]. This raises concern about the efficacy of prophylaxis with albendazole when immunosuppressive therapies are proposed for patients living in endemic areas. Treatment of strongyloidiasis is considered adequate only if the complete eradication of parasites occurs [13]. Thiabendazole has documented efficacy and has been demonstrated to clear the stool in most patients with strongyloidiasis [16]. Nevertheless, side effects are frequent with thiabendazole [16,17], reducing compliance to treatment. Ivermectin is usually much better tolerated and has recently become the drug of choice for treatment of Strongyloides infection. Compared to albendazole, ivermectin has shown better larval eradication rates 
[18]. Possibly, the failure of this prophylactic regimen is due to a lower efficacy of albendazole [18-20]; in the near future, more effective drugs, such as thiabendazole or ivermectin, should replace albendazole. We were not able to identify when these patients were infected, but disseminated strongyloidiasis occurred 10 days to seven months after the initiation of steroids and/or chemotherapy. Furthermore, in endemic areas, such as in Brazil, the possibility of reinfection should also be considered [21]. In patients receiving long-term immunosuppressive therapy and living in endemic areas, periodic antihelminthic prophylaxis may prove necessary. The exceedingly high mortality rate of disseminated strongyloidiasis in our series is a reminder of the fact that the use of traditional antihelminthic therapy (such as thiabendazole or ivermectin per os (PO) or enterally) may not be effective in this scenario. Abdominal distension is a prominent feature of patients presenting with disseminated strongyloidiasis (Table 2). This may result in inadequate biodisponibility of both thiabendazole and ivermectin, and consequently in poor therapeutic efficacy. Unfortunately, data on parenteral options for the treatment of disseminated strongyloidiasis are scarce. Recently, Chiodini et al. [22] reported successful subcutaneous administration of a veterinary preparation of ivermectin to control Strongyloides hyperinfection unresponsive to oral ivermectin and oral albendazole. However, solid data from clinical trials is necessary to advocate the use of parenteral ivermectin in humans.

In conclusion, we report a series of cases with purpuric periumbilical cutaneous lesions in the course of lethal disseminated strongyloidiasis. Careful observation of the first three cases allowed the recognition of purpuric lesions as a manifestation of disseminated strongyloidiasis and prompted anthelminthic treatment in the following cases. However, all of them developed MOF and died despite ICU support. Revision of the prophylactic and therapeutic regimens is recommended, considering the ominous prognosis of patients with disseminated infection.

\section{Acknowledgments}

We are indebted with Dr. Patricia Bozza for critical revision of this manuscript and to Dr. Paulo Faria for the histopathological evaluation.

\section{References}

1. Siddiqui A.A., Berk S.L. Diagnosis of Strongyloides stercoralis infection. Clin Infect Dis 2001;33:1040-7.

2. Stone W.J., Schaffner W. Strongyloides infections in transplant recipients. Semin Respir Infect 1990;5:58-64.

3. Ly M.N., Bethel S.L., Usmani A.S., Lambert D.R. Cutaneous Strongyloides stercoralis infection: an unusual presentation. J Am Acad Dermatol 2003;49(Suppl 2):S157-160

4. Purvis R.S., Beightler E.L., Diven D.G., et al. Strongyloides hyperinfection presenting with petechiae and purpura. Int J Dermatol 1992;31:169-71.

5. Reddy T.S. Syndrome of inappropriate secretion of antidiuretic hormone and nonpalpable purpura in a woman with Strongyloides stercoralis hyperinfection. Am J Med Sci 2003;325:288-91.

6. Ronan S.G., Reddy R.L., Manaligod J.R., et al. Disseminated strongyloidiasis presenting as purpura. J Am Acad Dermatol 1989;21:1123-5.

7. Kalb R.E., Grossman M.E. Periumbilical purpura in disseminated strongyloidiasis. JAMA 1986;256:1170-1.

8. von Kuster L.C., Genta R.M. Cutaneous manifestations of strongyloidiasis. Arch Dermatol 1988;124:1826-30.

9. Williams J., Nunley D., Dralle W., et al. Diagnosis of pulmonary strongyloidiasis by bronchoalveolar lavage. Chest 1988;94:643-4.

10. Mahmoud A.A.F. Strongyloidiasis. Clin Infect Dis 1996;23:949-52.

11. Genta R.M. Dysregulation of strongyloidiasis: a new hypothesis. Clin Microbiol Rev 1992;5:345-55.

12. Siddiqui A.A., Stanley C.S., Skelly P.J., Berk S.L. AcDNA encoding a nuclear hormone receptor of the steroid/ thyroid hormone-receptor superfamily from the human parasitic nematode Strongyloides stercoralis. Parasitol Res 2000;86:24-9.

13. Liu L.X., Weller P.F. Strongyloidiasis and other intestinal nematode infections. Infect Dis Clin North Am 1993;7:655-82.

14. Portugal R., Schaffel R., Almeida L., et al. Thiabendazole for the prophylaxis of strongyloidiasis in immunosuppressed patients with hematological diseases: a randomized double-blind placebo-controlled study. Haematologica 2002;87:663-4. 
15. Muenning P., Pallin D., Sell R.L., Chan M.-S. The cost effectiveness of strategies for the treatment of intestinal parasites in immigrants. New Engl J Med 1999;340:773-9.

16. Grove D.I. Treatment of strongyloidiasis with thiabendazole: an analysis of toxicity and effectiveness. Trans R Soc Trop Med Hyg 1982;76:114-8.

17. Gann P.H., Neva F.A., Gam A.A. A randomized trial of single- and two-dose ivermectin versus thiabendazole for treatment of strongyloidiasis. J Infect Dis 1994;169:1076-9.

18. Marti H., Haji H.J., Savioli L., et al. A comparative trial of a single-dose ivermectin versus three days of albendazole for treatment of Strongyloides stercoralis and other soil-transmitted helminth infections in children. Am J Trop Med Hyg 1996;55:477-81.

19. Liu L.X., Weller P.F. Antiparasitic drugs. N Engl J Med 1996;334:1178-84.

20. Keiser P.B., Nutman T.B. Strongyloides stercoralis in the immunocompromised population. Clin Microbiol Rev 2004;17:208-17.

21. Cimerman S., Cimerman B., Lewi D.S. Prevalence of intestinal parasitic infections in patients with acquired immunodeficiency syndrome in Brazil. Int J Infect Dis 1999;3:203-6.

22. Chiodini P.L., Reid A.J., Wiselka M.J., et al. Parenteral ivermectin in Strongyloides hyperinfection. Lancet 2000;355 43-4. 